\title{
DEGRADAÇÃO FOTOCATALÍTICA DE SULFAMETOXAZOL, TRIMETOPRIMA E DICLOFENACO EM SOLUÇÃO AQUOSA
}

\author{
Lutécia H. da Cruz, Fernanda G. Henning, Arlene B. dos Santos e Patricio Peralta-Zamora* \\ Departamento de Química, Universidade Federal do Paraná, CP 19081, 81531-990 Curitiba - PR, Brasil
}

Recebido em 14/8/09; aceito em 10/3/10; publicado na web em 23/6/10

\begin{abstract}
PHOTOCATALYTIC DEGRADATION OF SULFAMETOXAZOLE, TRIMETHOPRIM AND DICLOFENAC IN AQUOEUS SOLUTIONS. In this work the photocatalytic degradation of sulfametoxazole, trimethoprim and potassium diclofenac was evaluated by using $\mathrm{TiO}_{2}$ and $\mathrm{ZnO}$ photocatalysts. In optimized experimental conditions $\left(\mathrm{pH} 4, \mathrm{TiO}_{2}: 50 \mathrm{mg}\right)$ the $\mathrm{TiO}_{2}$-photocatalysis allowed an almost total degradation of the studied drugs with mineralization of about $80 \%$ at reaction times of $120 \mathrm{~min}$. Some mechanistic differences were observed between $\mathrm{TiO}_{2}$ and $\mathrm{ZnO}$ in the degradation study involving potassium diclofenac. At the first reaction times the use of $\mathrm{ZnO}$ leads to generation of transient species that strongly absorb in the UV spectral region, a fact not observed in studies involving $\mathrm{TiO}_{2}$.
\end{abstract}

Keywords: emerging pollutants; heterogeneous photocatalysis, drugs.

\section{INTRODUÇÃO}

Por muito tempo, os trabalhos desenvolvidos na área de tratamento de resíduos foram direcionados a um grupo de poluentes considerados prioritários, grande parte dos quais corresponde a espécies orgânicas resistentes e de acentuado caráter tóxico (ex. hidrocarbonetos policíclicos aromáticos e bifenilas policloradas). Nos últimos anos, entretanto, outros importantes micropoluentes e fontes de contaminação têm sido evidenciados, o que faz com que a disponibilização de sistemas de tratamento permaneça como importante desafio da área. Dentro do contexto dos poluentes emergentes, torna-se necessário salientar o grupo dos compostos que apresentam atividade farmacológica (PhACs - Pharmaceutically-active Compounds), os quais, em razão da massificação do seu uso e da baixa eficiência dos sistemas convencionais de tratamento de esgoto, contaminam águas superficiais e subterrâneas, provocando efeitos deletérios no meio hídrico e sérios problemas ambientais e de saúde pública. ${ }^{1-3}$ Uma interessante e completa revisão sobre a presença e o efeito deste tipo de substâncias no ambiente foi recentemente publicado por Bila e Dezotti. ${ }^{4}$

Em geral, estima-se que a maior fonte de contaminação por este tipo de compostos esteja representada pelas excreções metabólicas de pessoas em tratamento médico. Entretanto, uma grande quantidade destes PhACs pode ser liberada no ambiente em função de usos veterinários e da descarga de resíduos industriais e lixiviados de aterros sanitários ${ }^{5,6}$ Seja como for, a presença de antibióticos (como sulfametoxazol e trimetoprima), analgésicos, anti-inflamatórios (como diclofenaco) e hormônios tem sido relatada com bastante frequência em esgoto e águas naturais, ${ }^{7}$ o que representa um alerta para a necessidade de se desenvolver tecnologia de tratamento adequada.

De maneira geral, observa-se que processos convencionais de tratamento apresentam escassa utilidade na remoção deste tipo de poluentes. Nos processos biológicos, por exemplo, a eficiência de degradação é fortemente influenciada pela presença de outros macroconstituintes, o que faz com que a degradação dos fármacos, além de ocasional, seja apenas parcial. ${ }^{89}$ Sistemas fundamentados em processos de adsorção têm sido recentemente propostos, utilizandose sorbentes clássicos (carvão ativado) ${ }^{10}$ e modernos (micelas préadsorvidas em montmorilonita). ${ }^{11}$ Embora estes processos viabilizem

*e-mail: zamora@quimica.ufpr.br a depuração dos resíduos, o seu caráter não destrutivo implica na necessidade de procedimentos auxiliares, orientados ao tratamento ou à disposição das fases sólida em que os poluentes se encontram concentrados. Técnicas fundamentadas em processos de nanofiltração e osmose reversa costumam apresentar uma elevada eficiência na remoção de PhACs. ${ }^{12-14}$ Infelizmente, o elevado custo operacional envolvido dificulta o desenvolvimento de rotinas orientadas ao tratamento de grandes volumes de resíduos.

Na última década, inúmeros trabalhos têm demonstrado a excelente capacidade de degradação apresentada pela fotocatálise heterogênea frente a poluentes ambientais de relevância, ${ }^{15}$ principalmente utilizando-se dióxido de titânio $\left(\mathrm{TiO}_{2}\right)$ como fotocatalisador. ${ }^{16}$ Dentro deste contexto, pode ser dado destaque aos trabalhos recentemente apresentados por Klamerth e colaboradores ${ }^{17}$ e Abellán e colaboradores, ${ }^{18}$ que demonstram a eficiente degradação de fármacos, incluindo sulfametoxazol, trimetoprima e diclofenaco.

Embora o óxido de zinco $(\mathrm{ZnO})$ tenha apresentado melhor capacidade de degradação que o óxido de titânio frente a alguns poluentes ambientais, ${ }^{19}$ poucos trabalhos relatam o seu uso na degradação fotocatalítica de fármacos. Ate onde pudemos investigar, em apenas dois trabalhos se realizam estudos comparativos utilizando-se $\mathrm{TiO}_{2} \mathrm{e}$ $\mathrm{ZnO}$ (degradação de cloranfenicol ${ }^{20}$ e sulfametazina ${ }^{21}$ ), com resultados que indicam uma maior eficiência de degradação dos processos mediados por óxido de zinco.

O principal objetivo deste trabalho consistiu em estudar a degradação fotocatalítica de soluções aquosas contendo sulfametoxazol, trimetoprima e diclofenaco, utilizando-se $\mathrm{TiO}_{2}$ e $\mathrm{ZnO}$.

\section{PARTE EXPERIMENTAL}

\section{Reagentes}

Sulfametoxazol (All Chemistry do Brasil ${ }^{\circledR}, 99 \%$ de pureza), Trimetoprima (All Chemistry do Brasil ${ }^{\circledR}, 99 \%$ de pureza) e Diclofenaco Potássico (Importadora Deg ${ }^{\circledR}, 99 \%$ de pureza) foram utilizados em solução aquosa, em concentração de $20 \mathrm{mg} \mathrm{L}^{-1}$. A estrutura química destes fármacos é apresentada na Figura 1.

No processo de fotocatálise heterogênea foi utilizado dióxido de titânio Degussa P-25 (75\% anatase/25\% rutilo, BET $\left.50 \mathrm{~m}^{2} \mathrm{~g}^{-1}\right)$ e óxido de zinco Merck (wurtzita, BET $4 \mathrm{~m}^{2} \mathrm{~g}^{-1}$ ).

Outros reagentes foram de grau analítico PA. 


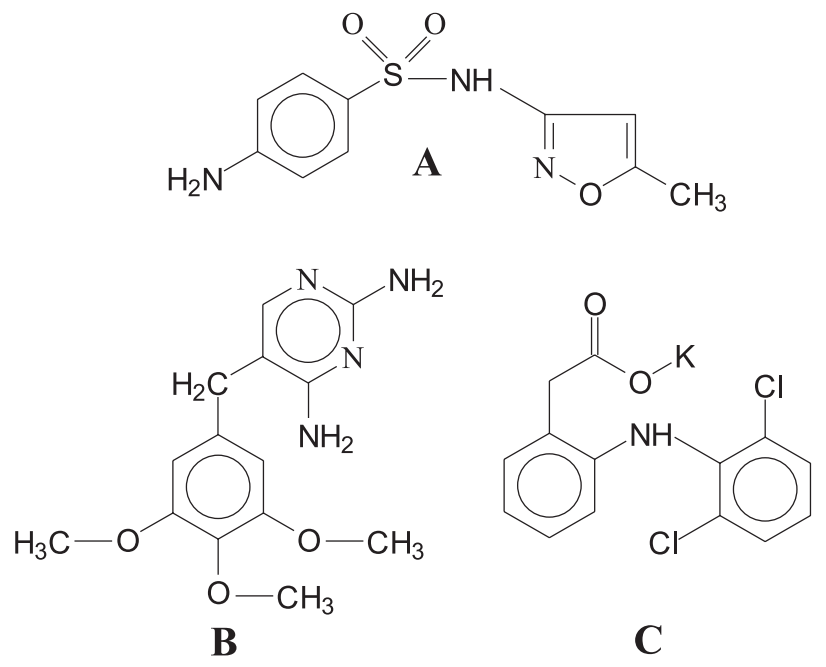

Figura 1. Estrutura química do sulfametoxazol (A), Trimetoprima (B) e diclofenaco potássico $(C)$

\section{Tratamento fotocatalítico}

Os estudos de degradação fotocatalítica foram conduzidos em um reator fotoquímico de bancada de $300 \mathrm{~mL}$ de capacidade, equipado com refrigeração por água e agitação magnética. A radiação ultravioleta foi proporcionada por uma lâmpada a vapor de mercúrio de $125 \mathrm{~W}$ (sem o bulbo protetor), inserida na solução por meio de um bulbo de quartzo (eficiência fotônica avaliada por actinometria (uranila/oxalato): $9,710^{-5} \mathrm{E} \mathrm{s}^{-1}$ ).

Neste reator, amostras de $250 \mathrm{~mL}$, em valores otimizados de $\mathrm{pH}$, foram adicionadas de quantidades otimizadas de semicondutor $\left(\mathrm{TiO}_{2}\right.$ e $\mathrm{ZnO}$ ) e borbulhadas com oxigênio comercial (vazão: $45 \mathrm{~mL}$ $\left.\min ^{-1}\right)$. Em todos os casos, alíquotas foram tomadas em intervalos regulares, filtradas em membrana de acetato de celulose $(45 \mu \mathrm{m}) \mathrm{e}$ submetidas à análise.

\section{Controle analítico}

Medidas espectrofotométricas foram realizadas em espectrofotômetro Shimadzu (2410 PC), utilizando cubetas de quartzo de $1 \mathrm{~cm}$ de caminho óptico. A degradação foi avaliada em função do decaimento da área espectral integrada, entre 190 e $350 \mathrm{~nm}$.

A determinação cromatográfica dos substratos em estudo foi realizada em cromatógrafo em fase líquida de alta eficiência Varian 920-LC, equipado com coluna C18 (microsorb-MV100-5, 250 x 4,6 $\mathrm{mm})$ e detector UV (254 nm). Para a determinação de sulfametoxazol e trimetoprima foi utilizada uma fase móvel constituída de acetonitrila (222 mL), água deionizada ( $777 \mathrm{~mL}$ ) e trietilamina (1,1 mL), com vazão de $1 \mathrm{~mL} \min ^{-1}$. Para a determinação de diclofenaco a fase móvel foi constituída por metanol:acetonitrila $(1: 1 \mathrm{v} / \mathrm{v}) \mathrm{em} \mathrm{pH} 5$ (tampão fosfato-

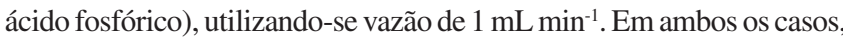
curvas analíticas individuais foram preparadas a partir de padrões puros (USP), na faixa de concentração compreendida entre 0,5 e $20 \mathrm{mg} \mathrm{L}^{-1}$, observando-se coeficientes de correlação $(r)$ superiores a 0,99 .

A determinação de carbono orgânico total foi realizada em um analisador de carbono orgânico total Shimadzu TOC-VCPH, fundamentado na oxidação catalítica a elevadas temperaturas e na determinação de $\mathrm{CO}_{2}$ por espectroscopia no infravermelho. Para o teor de carbono orgânico foram elaboradas duas curvas analíticas, a partir de um padrão de biftalato de potássio, cobrindo-se as faixas 0,5-5 e 5-200 $\mathrm{mg} \mathrm{L}^{-1}$. Para o carbono inorgânico o procedimento foi análogo, utilizando-se padrão misto de $\mathrm{Na}_{2} \mathrm{CO}_{3}$ e $\mathrm{NaHCO}_{3}$.

\section{RESULTADOS E DISCUSSÃO}

\section{Estudos preliminares}

Fotocatálise com $\mathrm{TiO}_{2}$

Inicialmente, o efeito de variáveis experimentais de relevância foi investigado, utilizando-se um sistema de planejamento fatorial de experimentos. Neste estudo, trimetoprima foi utilizada como substrato padrão, em função da maior resistência observada em estudos preliminares de degradação. O efeito das variáveis foi estudado em dois níveis, acrescidos de um ponto central ensaiado em triplicata (pH: 4,0, 6,0 e 8,0; concentração de fotocatalisador: 200, 300 e 400 $\left.\mathrm{mg} \mathrm{L}^{-1}\right)$, o que configura um planejamento $2^{2}$.

Os resultados são apresentados na forma de uma representação geométrica (Figura 2), considerando-se como resposta a diminuição percentual do teor de carbono orgânico total, para tempos de reação de $60 \mathrm{~min}$. O efeito positivo da concentração de fotocatalisador (5+/-2) indica que processos de degradação são favorecidos quando maiores massas de fotocatalisador são utilizadas, enquanto que o efeito negativo do $\mathrm{pH}(-15+/-2)$ indica uma maior eficiência de degradação em valores de $\mathrm{pH}$ próximos ao mínimo estudado $(4,0)$. Entretanto, um significativo efeito de segunda ordem (12+/-2) indica a existência de importantes efeitos de interação, o que diminui a importância dos efeitos isolados e obriga a uma análise dos resultados em conjunto.

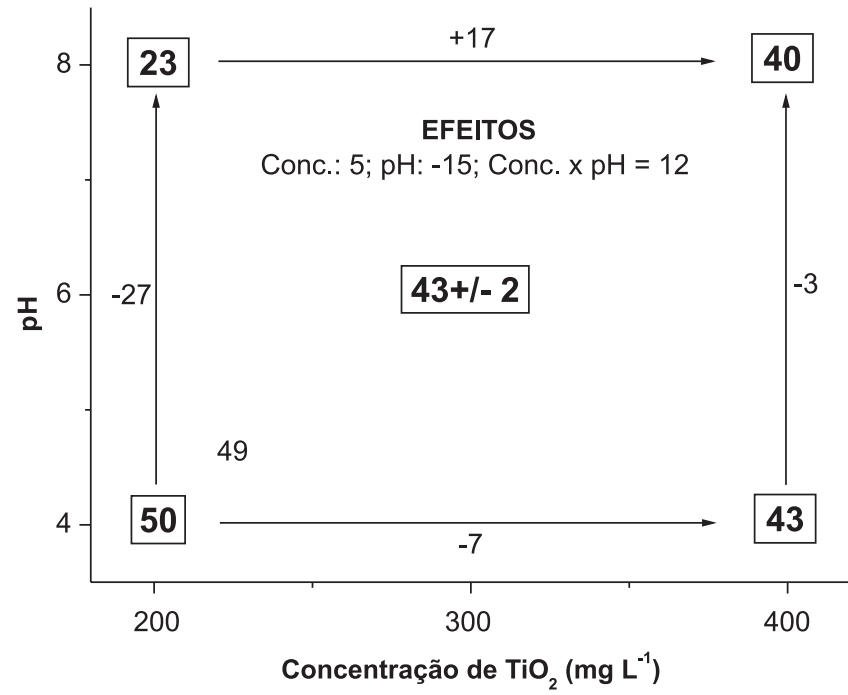

Figura 2. Representação geométrica do planejamento fatorial $2^{2}$ utilizado para investigação do efeito das variáveis concentração de $\mathrm{TiO}_{2}$ e pH na degradação fotocatalítica de trimetoprima (trimetoprima: $20 \mathrm{mg} \mathrm{L}^{-1}$, volume: $250 \mathrm{~mL}$, tempo de reação: 60 min, Resposta: redução do teor de carbono orgânico total)

Levando em consideração o desvio médio observado na avaliação das respostas (+/-2) é possível admitir que as maiores taxas de mineralização foram obtidas em $\mathrm{pH}$ entre 4 e 6 , com pouca influência da concentração do fotocatalisador. O efeito do $\mathrm{pH}$ é de difícil interpretação, em função de influenciar tanto o equilíbrio de protonação do substrato, como as propriedades superficiais do catalisador. $\mathrm{TiO}_{2}$ apresenta um caráter anfótero, com ponto de carga zero em $\mathrm{pH}$ 6,25.22 Assim, em valores inferiores de $\mathrm{pH}$ a sua superfície tende a ficar positivamente carregada, o que pode favorecer a adsorção de substratos que apresentam a função amina, como a trimetoprima. Antecedentes demonstram que em valores de $\mathrm{pH}$ entre 3,5 e 5,5 a adsorção de algumas aminas é maximizada, em função de interações entre a superfície positiva do catalisador e o par de elétrons nãoligantes do nitrogênio amino. ${ }^{23}$ 
Em função destas observações, estudos subsequentes foram realizados em $\mathrm{pH} 4,0$, utilizando-se o fotocatalisador em concentração de $200 \mathrm{mg} \mathrm{L}^{-1}$. Nestas condições, a degradação se processa de forma rápida, o que provoca significativas modificações espectrais nos primeiros tempos monitorados (Figura 1S, material suplementar). Após 30 min de reação, o monitoramento cromatográfico mostra concentrações de trimetoprima inferiores ao limite da quantificação $\left(0,5 \mathrm{mg} \mathrm{L}^{-1}\right)$, o que implica em remoções superiores a $97,5 \%$ (Figura 3 ). A permanência de sinais na região centrada entre 200 e $226 \mathrm{~nm}$, mesmo na presença de baixas concentrações de trimetoprima, confirma a formação de espécies transientes que mantêm o caráter aromático, as quais, entretanto, são também degradadas em maiores tempos de reação. A mineralização da ordem de $80 \%$ e a presença de intensos sinais residuais entre 190 e $200 \mathrm{~nm}$ sugerem o acúmulo de produtos de degradação de baixa massa molecular, como alcoóis, aldeídos e ácidos carboxílicos, tal como reportado em estudos de degradação envolvendo sulfametoxazol. ${ }^{24}$

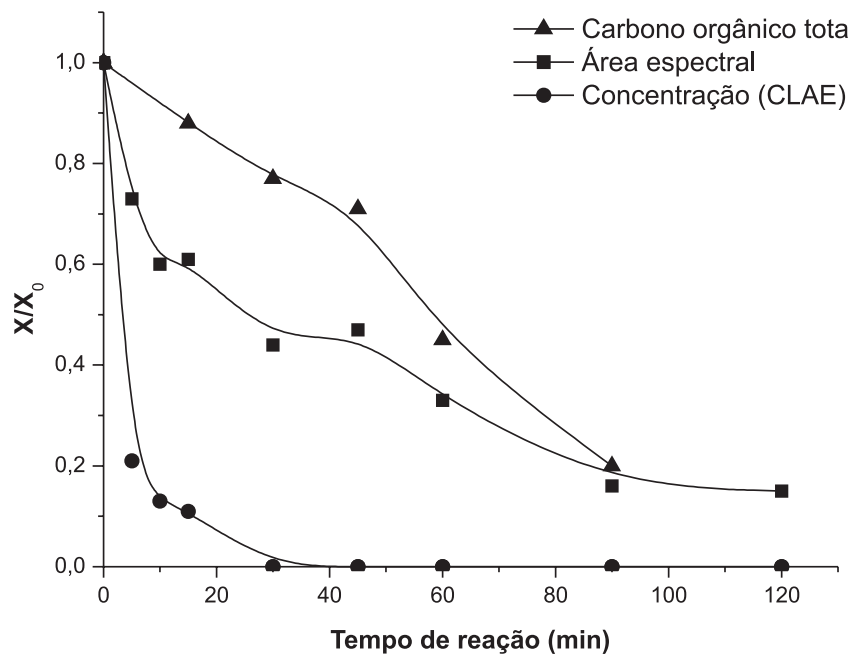

Figura 3. Modificação dos parâmetros de área espectral, carbono orgânico total e concentração de trimetoprima por CLAE durante tratamento fotocatalítico de trimetoprima utilizando-se suspensões de $\mathrm{TiO}_{2}$. (trimetoprima: 20 $m g L^{-1}$, volume: $250 \mathrm{~mL}, \mathrm{pH}: 4,0, \mathrm{TiO}_{2}: 200 \mathrm{mg} \mathrm{L}^{-1}$ )

\section{Fotocatálise com $\mathrm{ZnO}$}

O estudo preliminar, orientado a avaliar o efeito da concentração do fotocatalisador e do $\mathrm{pH}$ da solução na eficiência da degradação de trimetoprima, permitiu a obtenção dos resultados apresentados na Figura 4. Estes resultados demonstram um efeito pouco significativo da concentração $(0,5+/-3)$ e um efeito negativo do $\mathrm{pH}(-6,5+/-3)$, resultado, este último, que indica melhores condições de degradação em $\mathrm{pH}$ próximo de 4.

Assim como o $\mathrm{TiO}_{2}$, óxido de zinco apresenta características anfóteras, com ponto de carga zero em $\mathrm{pH}$ próximo de $8 .{ }^{25}$ Assim, em valores inferiores a este limite a superfície do catalisador tende a ficar positivamente carregada, o que pode favorecer a adsorção de substratos como a trimetoprima e, consequentemente, a sua degradação fotocatalítica.

Trabalhando nas condições de máxima eficiência de degradação ( $\mathrm{pH}$ 4,0, concentração de $\mathrm{ZnO}$ de $200 \mathrm{mg} \mathrm{L}^{-1}$ ) obtiveram-se os resultados apresentados nas Figuras 2S, material suplementar, e 5. Na sequência de espectros apresentados na Figura $2 \mathrm{~S}$ é possível observar uma rápida degradação de trimetoprima, o que permite a obtenção de um perfil espectral significativamente diferenciado em tempos de reação de $60 \mathrm{~min}$. Embora a presença do substrato em estudo não

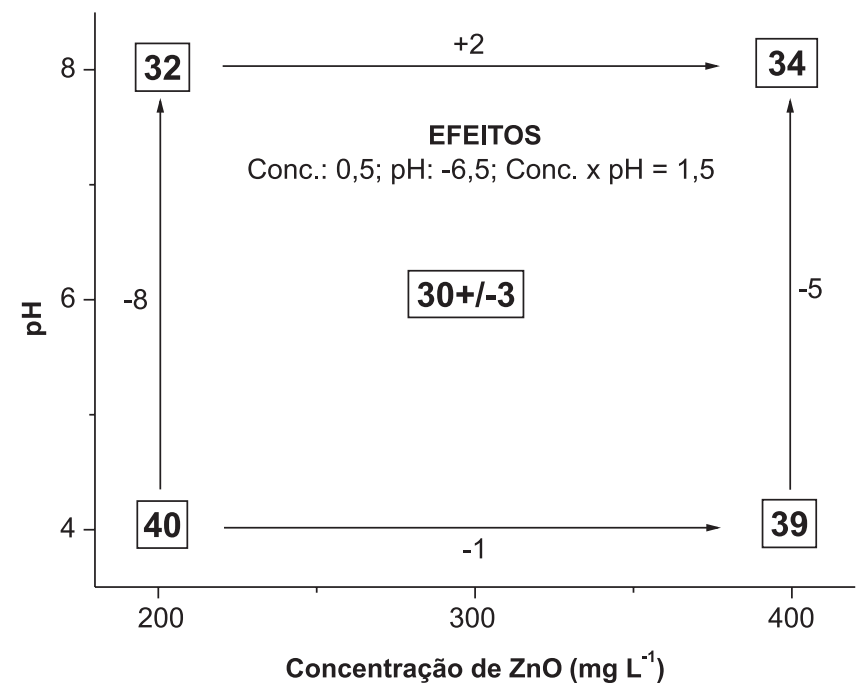

Figura 4. Representação geométrica do planejamento fatorial $2^{2}$ utilizado para investigação do efeito das variáveis concentração de $\mathrm{ZnO}$ e pH na degradação fotocatalítica de trimetoprima (trimetoprima: $20 \mathrm{mg} \mathrm{L}^{-1}$, volume: $250 \mathrm{~mL}$, tempo de reação: 60 min, Resposta: redução do teor de carbono orgânico total)

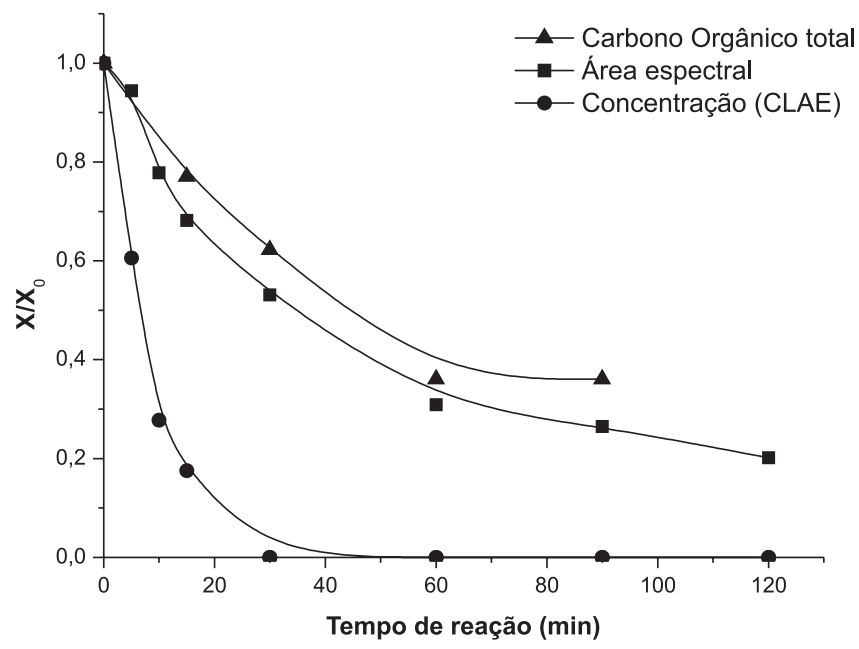

Figura 5. Modificação dos parâmetros de área espectral, carbono orgânico total e concentração de trimetoprima por CLAE (B), durante tratamento fotocatalítico de trimetoprima utilizando-se suspensões de $\mathrm{ZnO}$. (trimetoprima: $20 \mathrm{mg} \mathrm{L}^{-1}$, volume: $250 \mathrm{~mL}$, $\mathrm{pH}: 4,0, \mathrm{ZnO}: 200 \mathrm{mg} \mathrm{L}^{-1}$ )

tenha sido cromatograficamente detectada a partir de tratamentos de 30 min (Figura 5), a persistência de sinais espectrais e a observação de taxas de mineralização máximas de $60 \%$ indicam o acúmulo de intermediários, provavelmente sem caráter aromático.

\section{Estudos de degradação de trimetoprima, sulfametoxazol e diclofenaco}

Inicialmente, pretendia-se a realização de uma comparação quantitativa entre os processos envolvendo $\mathrm{TiO}_{2}$ e $\mathrm{ZnO}$, utilizandose parâmetros cinéticos do processo de degradação. Infelizmente, a rápida degradação dos substratos impediu a obtenção de dados cromatográficos em número suficiente, o que fez com que se selecionasse a redução da área espectral integrada como parâmetro de comparação.

Os resultados (Figura 6A) indicam uma maior eficiência de degradação do sistema fotoquímico operado com dióxido de titânio, o qual, com exceção de trimetoprima, permitiu uma eliminação pra- 
ticamente completa dos sinais espectrais monitorados, em tempos de reação de $30 \mathrm{~min}$. Tal como comentado anteriormente, a trimetoprima apresentou-se mais resistente, precisando de tempos de tratamento significativamente maiores. Adicionalmente, a fotocatálise mediada por $\mathrm{TiO}_{2}$ permitiu mineralizações superiores a $80 \%$, nos maiores tempos de reação praticados (120 min).
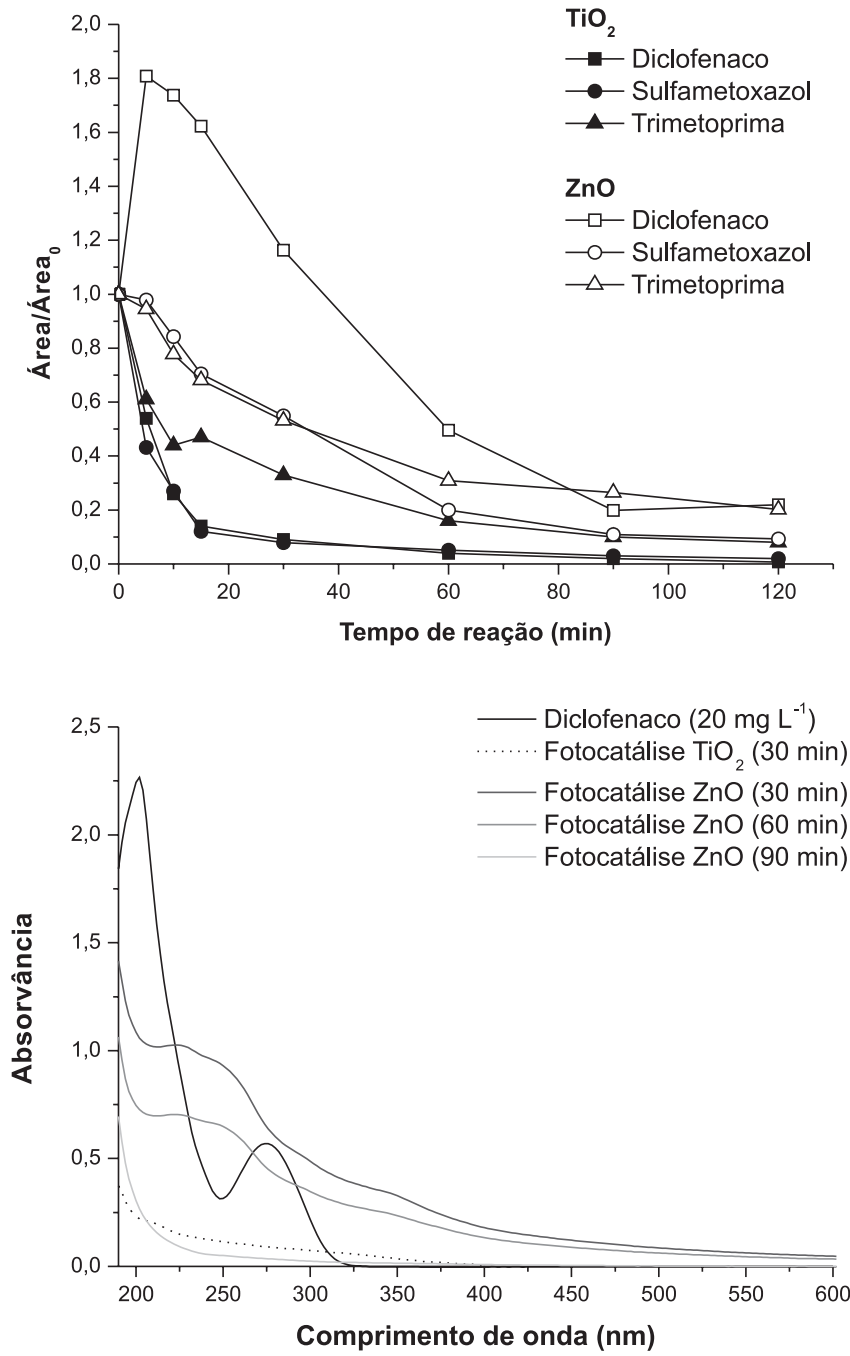

Figura 6. Modificação da área espectral (área/Área ${ }_{0}$ ) durante a degradação fotocatalítica de trimetoprima, sulfametoxazol e diclofenaco utilizando-se suspensões de $\mathrm{TiO}_{2}$ e $\mathrm{ZnO}$ (A) e perfil espectral de soluções aquosas de diclofenaco tratadas por fotocatálise com $\mathrm{TiO}_{2}$ e $\mathrm{ZnO}$ (B). (fármaco: $20 \mathrm{mg}$ $L^{-1}$, volume: $250 \mathrm{~mL}, \mathrm{pH}: 4,0$, Fotocatalisador: $200 \mathrm{mg} \mathrm{L}^{-1}$ )

O processo de degradação mediado por óxido de zinco mostrou-se menos eficiente, precisando de maiores tempos de reação para produzir efeitos similares aos provocados pelo $\mathrm{TiO}_{2}$. Neste caso, mineralizações da ordem de 50 a $70 \%$ foram observadas em 120 min de tratamento.

Além das diferenças observadas na eficiência de degradação, importantes diferenças mecanísticas foram observadas no tratamento de diclofenaco de potássio (Figura 6B); enquanto o processo assistido por $\mathrm{TiO}_{2}$ provocou uma redução sistemática da área espectral, o óxido de zinco induziu um aumento do sinal espectral nos primeiros tempos monitorados ( $5 \mathrm{~min}$ ), sinal que, entretanto, foi reduzido nos maiores tempos de reação. Estudos de degradação de diclofenaco envolvendo o uso de $\mathrm{TiO}_{2}$ sugerem a rápida geração de formas hidroxiladas do substrato, as quais, após fragmentação, levam à formação de espécies como diclorofenol, clorocatecol, catecol e hidroquinona. ${ }^{26}$
A ausência de trabalhos que envolvam a degradação de diclofenaco por fotocatálise mediada por $\mathrm{ZnO}$ impede uma análise aprofundada desta observação. Entretanto, a identificação de intensos sinais de absorção centrados em 222, 242, 288 e 339 nm sugere a formação preliminar de espécies de maior absortividade, o que, por sua vez, confirma a existência de mecanismos de degradação diferenciados. Em estudos de degradação envolvendo 2-fenil-fenol, Khodja e colaboradore ${ }^{27}$ confirmaram a presença de hidroquinona e benzoquinona como principais intermediários de degradação, na presença de $\mathrm{ZnO}$. $\mathrm{Na}$ presença de $\mathrm{TiO}_{2}$ estes intermediários aparecem em nível de traço, o que demonstra a existência de mecanismos diferenciados.

\section{CONCLUSÕES}

Processos de fotocatálise heterogênea permitem uma eficiente degradação dos fármacos em solução aquosa, reduzindo a sua concentração até níveis não detectáveis por cromatografia $\left(0,5 \mathrm{mg} \mathrm{L}^{-1}\right)$ em tempos de reação de 30 (trimetoprima) e 10 min (diclofenaco e sulfametoxazol).

Dióxido de titânio proporciona degradações mais favoráveis, permitindo mineralizações da ordem de $80 \%$ em 120 min de tratamento.

Diferenças observadas no comportamento espectral das amostras de diclofenaco sugerem diferenças mecanísticas entre os processos mediados por $\mathrm{TiO}_{2} \mathrm{e} \mathrm{ZnO}$, o que leva à geração de espécies transientes diferentes nos primeiros minutos de reação.

\section{MATERIAL SUPLEMENTAR}

Está disponível em http://quimicanova.sbq.org.br, na forma de arquivo .PDF, com acesso livre. Nas Figuras $1 \mathrm{~S}$ e $2 \mathrm{~S}$ apresenta-se a modificação do perfil espectral durante o tratamento fotocatalítico de trimetoprima utilizando-se suspensões de $\mathrm{TiO}_{2}$ e $\mathrm{ZnO}$, respectivamente.

\section{REFERÊNCIAS}

1. Kim, J. H.; Park, P. K.; Lee, C. H.; Kwon, H. H.; J. Membr. Sci. 2008, $321,190$.

2. Radjenović, J.; Petrović, M.; Barceló, D.; Water Res. 2009, 43, 831.

3. Kimura, K.; Hara, H.; Watanabe, Y.; Desalination 2005, 178, 135.

4. Bila, D. M.; Dezotti, M.; Quim. Nova 2003, 26, 523.

5. Heberer, T.; Toxicol. Lett. 2002, 131, 5.

6. Bendz, D.; Paxéus, N. A.; Ginn, T. R.; Loge, F. J.; J. Hazard. Mater. 2005, 122, 195

7. Hernando, M. D.; Mezcua, M.; Fernández-Alba, A. R.; Barceló, D.; Talanta 2006, 69, 334.

8. Joss, A.; Zabczynski, S.; Göbel, A.; Hoffmann, B.; Löffler, D.; Mcardell, C. S.; Ternes, T. A.; Thomsen, A.; Siegrist, H.; Water Res. 2006, 40, 1686.

9. Drillia, P.; Dokianakis, S. N.; Fountoulakis, M. S.; Kornaros, M.; Stamatelatou, K.; Lyberatos, G.; J. Hazard. Mater. 2005, 122, 259.

10. Casas, R. N.; Rodriguez, A. G.; Bueno, F. R.; Lara, A. E.; Calahorro, C. V.; Guijosa, A. N.; Appl. Surf. Sci. 2006, 252, 6022.

11. Polubesova, T.; Zadaka, D.; Groisman, L.; Nir, S.; Water Res. 2006, 40, 2369.

12. Yoon, Y.; Westerhoff, P.; Snyder, S. A.; Wert, E. C.; Yoon, J.; Desalination 2007, 202, 16.

13. Comerton, A. M.; Andrews, R. C.; Bagley, D. M.; Hao, C.; J. Membr. Sci. 2008, 313, 323.

14. Hua, W.; Bennett, E. R.; Letcher, R. J.; Water Res. 2006, 40, 2259.

15. Fujishima, A.; Zhang, X.; Tryk, D. A.; Int. J. Hydrogen Energy 2007 , 32, 2664.

16. Fujishima, A.; Rao, T. N.; Tryk, D. A.; J. Photochem. Photobiol., C $2000,1,1$. 
17. Klamerth, N.; Malato, M. S.; Agüera, A.; Fernández-Alba, A. R.; Maldonado, M. I.; Coronado, J. M.; Catal. Today 2009, 144, 124.

18. Abellán, M. N.; Giménez, J.; Esplugas, S.; Catal. Today 2009, 144, 131.

19. Hariharan, C.; Appl. Catal., A 2006, 304, 55.

20. Chatzitakis, A.; Berberidou, C.; Paspaltsis, I.; Kyriakou, G.; Sklaviadis, T.; Poulios, I.; Water Res. 2008, 42, 386.

21. Kaniou, S.; Pitarakis, K.; Barlagianni, I.; Poulios, I.; Chemosphere 2005, $60,372$.

22. Son, H. S.; Lee, S. J.; Cho, I. H.; Zoh, K. D.; Chemosphere 2004, 57, 309.
23. Canle, M. L.; Santaballa, J. A.; Vulliet, E.; J. Photochem. Photobiol., A 2005, 175, 192.

24. Hu, L.; Flanders, P. M.; Miller, P. L.; Strathmann, T. J.; Water Res. 2007, $41,2612$.

25. Rao, A. N.; Sivasankar, B.; Sadasivam, V.; J. Hazard. Mater. 2009, 166 , 1357.

26. Calza, P.; Sakkas, V. A.; Medana, C.; Baiocchi, C.; Dimou, A.; Pelizzetti, E.; Albanis, T.; Appl. Catal., B 2006, 67, 197.

27. Khodja, A. A.; Sehili, T.; Pilichowski, J. F.; Boule, P.; J. Photochem. Photobiol., A 2001, 141, 239. 
Lutécia H. da Cruz, Fernanda G. Henning, Arlene B. dos Santos e Patricio Peralta-Zamora*

Departamento de Química, Universidade Federal do Paraná, CP 19081, 81531-990 Curitiba - PR, Brasil

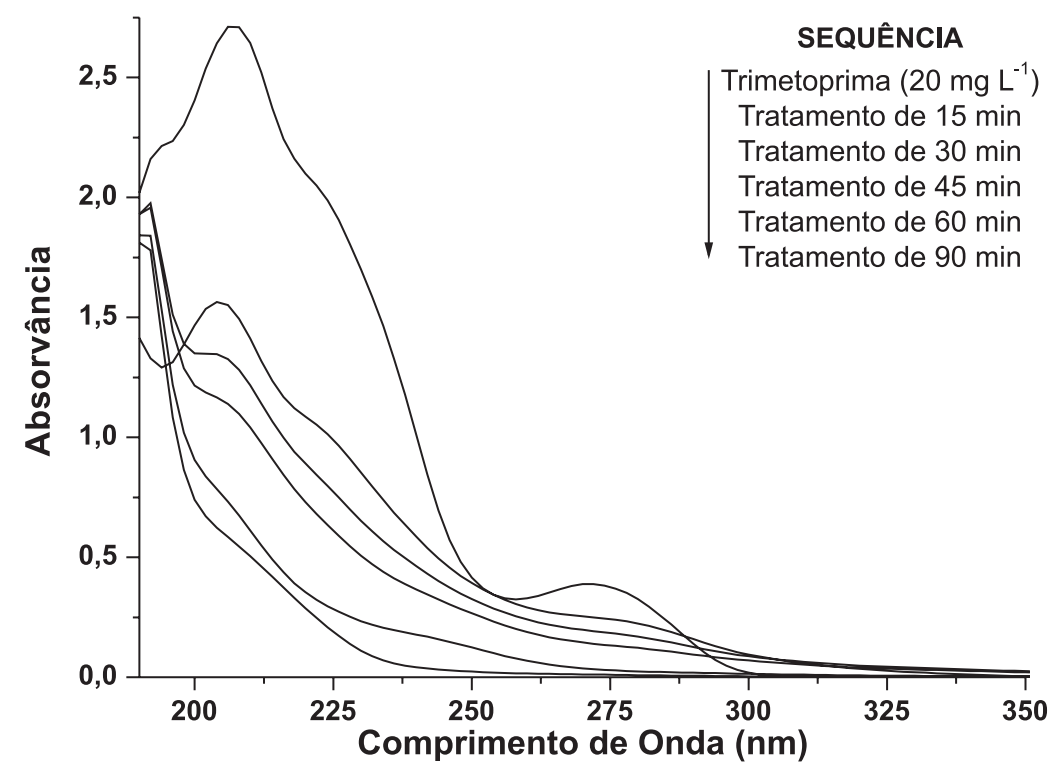

Figura 1S. Modificação do perfil espectral durante o tratamento fotocatalítico de trimetoprima utilizando-se suspensões de $\mathrm{TiO}_{2}$. (trimetoprima: $20 \mathrm{mg} \mathrm{L}^{-1}$, volume: $250 \mathrm{~mL}, \mathrm{pH}: 4,0, \mathrm{TiO}_{2}: 200 \mathrm{mg} \mathrm{L}^{-1}$ )

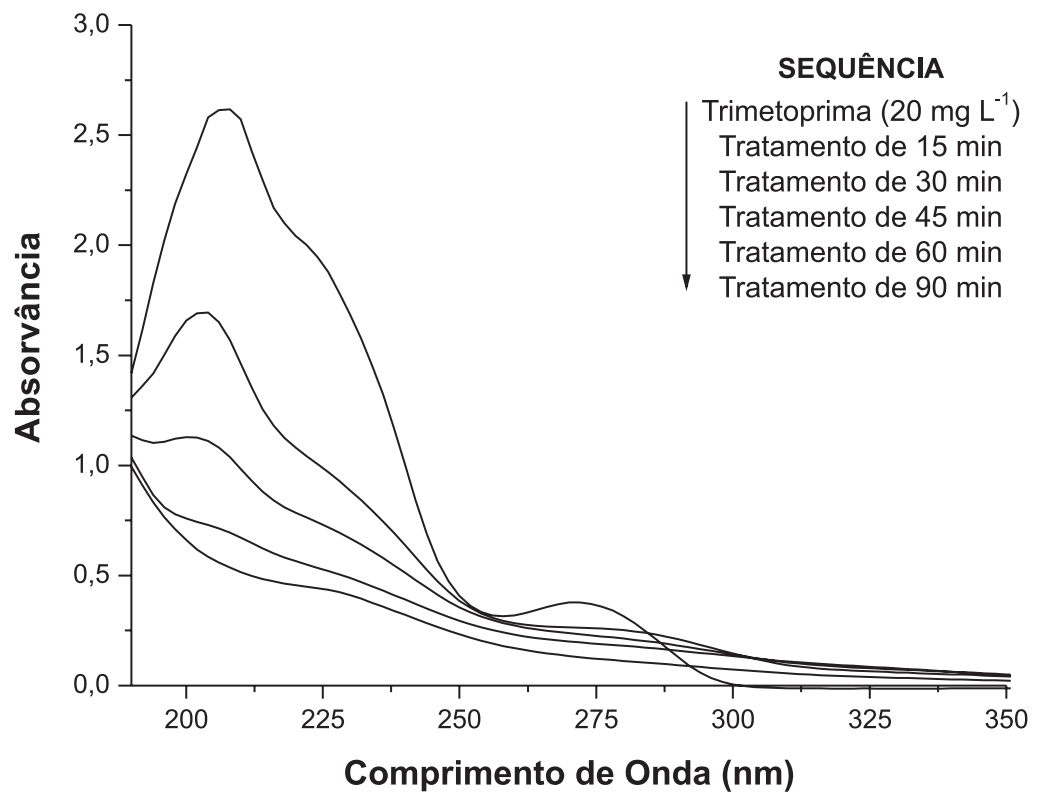

Figura 2S. Modificação do perfil espectral durante o tratamento fotocatalítico de trimetoprima utilizando-se suspensões de ZnO. (trimetoprima: 20 mg $L^{-1}$, volume: $250 \mathrm{~mL}, \mathrm{pH}: 4,0, \mathrm{ZnO}: 200 \mathrm{mg} \mathrm{L}^{-1}$ ) 